\title{
Alternative Integrated Testing for Skin Sensitization: Assuring Consumer Safety
}

\author{
Aurelia Del Bufalo,' Thierry Pauloin,, Nathalie Alepee,, Jacques Clouzeau, Ann Detroyer, \\ Joan Eilstein, Charles Gomes, Hicham Nocairi, Cécile Piroird, Francoise Rousset, \\ Fleur Tourneix, David Basketter, and Silvia Martinozzi Teissier ${ }^{1}$
}

\begin{abstract}
Cosmetics legislation in Europe has driven the validation and acceptance of non-animal alternatives, most recently in the area of skin sensitization. Despite use of these methods to meet regulatory needs, it is also essential that they allow evaluation regarding human safety. For cosmetic product safety, it is necessary to understand how they can be used and with what limitations, and thereby reveal what remains to be addressed. A dataset of 165 ingredients (137 cosmetic ingredients +28 reference substances) has been identified, curated, and subjected to testing using accepted in vitro methods, with additional information, including physicochemical data and in silico results. The inputs from multiple determinants of skin sensitizing activity have been used in five individual supervised classification models (or machine learning approaches), which were then collated in a robust statistical manner, a stacking meta-model, to deliver a prediction with an optimized level of confidence. For the training set, with the probability cutoffs at $70 \%$ and $30 \%$, predictive sensitivity was $97 \%$, specificity was $88 \%$, the overall accuracy was $93 \%$, and kappa was $85 \%$. A further 52 substances were used to test the effectiveness of the model: the predictive sensitivity was $89 \%$, specificity was $95 \%$, overall accuracy was $91 \%$, and kappa was $82 \%$. In conclusion, this stacking meta-model delivers improved performance and therefore enhanced confidence in the discrimination of skin sensitizers from nonsensitizers. The key remaining gap, prediction of skin sensitization potency, may benefit from a similar approach, maximizing use of evidence from individual strands of prediction, while minimizing the impact of the limitations from any particular one.
\end{abstract}

Keywords: skin sensitization, in vitro tests, in silico, confidence estimation, integrated testing strategy, risk assessment, consumer safety, cosmetics

\section{Introduction}

$\mathbf{T}$ HE REPLACEMENT OF IN VIVO ASSAYS by non-animal alternatives has been a key goal for toxicologists for more than 50 years. ${ }^{1}$ Following the principles outlined, legislation in the European Union (EU) has long required that the use of in vivo assays was a last resort, with the details being updated relatively recently. ${ }^{2}$ However, specifically for cosmetic products, a comprehensive ban on the use of animal testing was foreseen by EU legislation and came fully into force in March 2013. ${ }^{3}$ At the same time, it remained, and remains, both a legal and a moral responsibility that cosmetic products should be safe for the consumer. ${ }^{4,5}$ Driven by both ethical considerations and legislative requirements in Europe and elsewhere, approaches to toxicology testing that avoid entirely the use of animals have been a goal for several decades. ${ }^{6,7}$ For the toxicology endpoint of skin sensitization, the predictive identification, characterization, and assessment have progressed from the use of guinea pigs, ${ }^{8,9}$ through more refined use of a validated murine assay, ${ }^{10}$ to the present situation involving a suite of non-animal alternatives. ${ }^{11,12}$ These transitions not only brought significant benefits but also presented some important challenges.

The formalization of the adverse outcome pathway (AOP),${ }^{13,14}$ including the identification of the key events in the mechanism of skin sensitization, facilitated the processes associated with validation and acceptance on non-animal alternatives. What followed quickly was the successful validation of three in vitro skin sensitization methods (the direct peptide reactivity assay-DPRA, KeratinoSens ${ }^{\mathrm{TM}}$, and the human cell line activation test-h-CLAT) and their adoption into OECD test guidelines. Thus the goal for the replacement

\footnotetext{
${ }^{1}$ L'Oréal R\&I, Aulnay-sous-Bois, France.

${ }^{2}$ DABMEB Consultancy Ltd., Sharnbrook, United Kingdom.
}

(C) Aurelia Del Bufalo et al., 2018; Published by Mary Ann Liebert, Inc. This is an Open Access article distributed under the terms of the Creative Commons Attribution License, which permits unrestricted use, distribution, and reproduction in any medium, provided the original work is properly cited. 
of in vivo assays by non-animal alternatives appears to have been reached. ${ }^{15-17}$ In addition, several further methods are in the final stages of review at the OECD, including U-SENS $^{\text {TM }}{ }^{18,19}$ (www.oecd.org/env/ehs/testing/TG-Usensdraft-TG_Dec15-2016-clean.pdf) and IL8 Luc ${ }^{20}$ (www1.oecd .org/env/ehs/testing/Draft-IL-8-Luc-TG-15Dec_clean.pdf), or under consideration, including LuSens ${ }^{21}$ and SENS-IS. ${ }^{22}$ Although there remains some debate, the current consensus holds that none of the methods in isolation is sufficient for hazard identification, which means that strategies to combine outputs from these methods must be developed. ${ }^{23-25}$ Toward this end have been directed a range of activities/ proposals with their associated publications. ${ }^{11,26-30}$ Indeed, for the primary step of the identification of skin sensitization hazard in the context of regulations such as REACH in Europe, a simple majority verdict based on "yes/no" outputs from these assays has been deemed by many to be equivalent, or even better, than the Local Lymph Node Assay (LLNA). ${ }^{1,29,31,32}$

Obviously, this progress in the avoidance of animal testing for the skin sensitization end point is laudable for many reasons. When a substance is identified as a "nonsensitizer," the level of uncertainty associated with that determination is by no means definitive. In principle, it should be possible to use a truly nonsensitizing cosmetic ingredient in any product type and at any desired level (subject of course to an absence of toxic effects other than skin sensitization). However, this can only be done with equanimity if there is a high level of confidence in the prediction that the ingredient is truly nonsensitizing.

In light of these perspectives, we have adopted an approach based on practical application of published statistical tools for the optimized combination of multiple predictive assays. ${ }^{33,34}$ A condensed and preliminary version of this work was shared with the OECD to assist in their program concerning integrated approaches to testing and assessment ${ }^{24}$; in this article, we present an updated and completed version of our work.

\section{Materials and Methods}

\section{Data sources}

Material has been derived from both the published literature (72 substances) and internal studies conducted by L'Oréal Laboratories on proprietary materials (93 substances). Details of the 165 substances used for the training $(n=113)$ and the test $(n=52)$ datasets are presented in Tables 1 and 2. Note that for the proprietary materials, the CAS number has been replaced by a L'Oréal internal coding number. Chemicals were randomly allocated to each set, by a process that was repeated until the training and test datasets were balanced as far as possible regarding the proportion of sensitizers (S) and nonsensitizers (NS). In Figure 1, several aspects (cosmetic classes, sources of data, and so on) of the complete chemical set are presented, illustrating the fact that the set is quite balanced, including the LLNA potency used to define potency subcategories Figure $1 \mathrm{~A}$ and $\mathrm{B}$. This balance between $\mathrm{S}$ and NS has been maintained in the training set (47 NS and $66 \mathrm{~S}$ ) and in the test set (21 NS and $31 \mathrm{~S}$ ).

Subsequently, a number of inputs were selected, those aiming to ensure proper coverage of the key events of the AOP using validated tests (DPRA, KeratinoSens, and U-SENS), as well as the most statistically impactful physicochemical parameters, $\mathrm{pH}$ and volatility, ${ }_{35}$ together with in silico pre- dictions (ToxTree and TIMES-SS), the latter including aspects of metabolic activation. Each of these is detailed below.

\section{Physicochemical data}

Volatility was assessed using the MPBPWIN ${ }^{\mathrm{TM}}$ model from the open source Episuite ${ }^{\mathrm{TM}}$ software. ${ }^{36}$ Based on the structure of a given chemical, the model estimates vapor pressure (VP) from various physicochemical equations. These values were converted into volatility classes ${ }^{37}$ : VP $<10^{-7} \mathrm{mmHg}=$ non volatile; VP between $10^{-7}$ and $10^{-1}$ $\mathrm{mmHg}=$ semi volatile; VP between $10^{-1}$ and $380 \mathrm{mmHg}=$ volatile; and $\mathrm{VP}>380 \mathrm{mmHg}=$ very volatile. Pragmatically, these last two groups were grouped together into a "very volatile" class. All $\mathrm{pH}$ values were obtained using a method adapted from OECD Guideline for the Testing of Chemicals No. $122 .{ }^{38}$

\section{In silico inputs}

ToxTree, an open-source in silico prediction software from Ideaconsult Ltd containing skin sensitization alerts based on the reaction mechanistic domains classification, was used (http://toxtree.sourceforge.net). Its "Skin Sensitization Alerts" decision tree using "SMARTS," which relies on a Reaction Mechanistic Domains classification, delivers alerts for a parent chemical structure. These alerts are transparent as for each chemical, the applied decision rules are shown in the software's interface. Where nucleophilic aromatic substitution $\left(\mathrm{S}_{\mathrm{N}} \mathrm{Ar}\right)$, bimolecular nucleophilic substitution $\left(\mathrm{S}_{\mathrm{N}} 2\right)$, acyl transfer agent, Michael acceptor, and Schiff base formation alerts are identified, the chemical is classified as reactive (sensitizer), whereas in the absence of reactivity alerts, it is classified as nonreactive (nonsensitizer) ${ }^{39}$

The in silico TIssue MEtabolism Simulator (TIMES-SS) from OASIS-LMC (http://oasis-lmc.org/products/software/ times.aspx), integrating the Skin Sensitization prediction model, analyzes the parent molecular structure as well as its simulated metabolites to predict skin sensitization potency based on structural alerts and 3D-QSARs. For each chemical, detailed information on the applied mechanistic structural alerts and metabolite simulation is available in the software's interface. The overall "in domain" prediction that is retained is that of the most potent structure among parent and metabolites, and is expressed as one of following three classes: nonsensitizer, weak sensitizer, or strong sensitizer. ${ }^{40}$ For this work, weak and strong sensitizers have been grouped together [as hereafter identified as positive (+)].

\section{In vitro methods}

The DPRA ${ }^{15}$ represents key event 1 on the skin sensitization AOP. ${ }^{13,14}$ The reactivity assay DPRA is based on the depletion measurement of two synthetic peptides (containing either cysteine or lysine amino acids) after reaction with the test chemical. Chemicals that induce peptide depletion above a defined threshold are considered to be reactive and thus give a prediction. ${ }^{41}$ If the reactivity is below this level, the prediction is negative. Sometimes, the result is regarded as inconclusive, due to the co-elution of both synthetic peptides and the test material.

The KeratinoSens assay ${ }^{16}$ models key event 2 of the AOP by measuring the activation of the Nrf2-Keap-1 pathway, the cellular sensor of electrophilic/redox stress, which is induced 


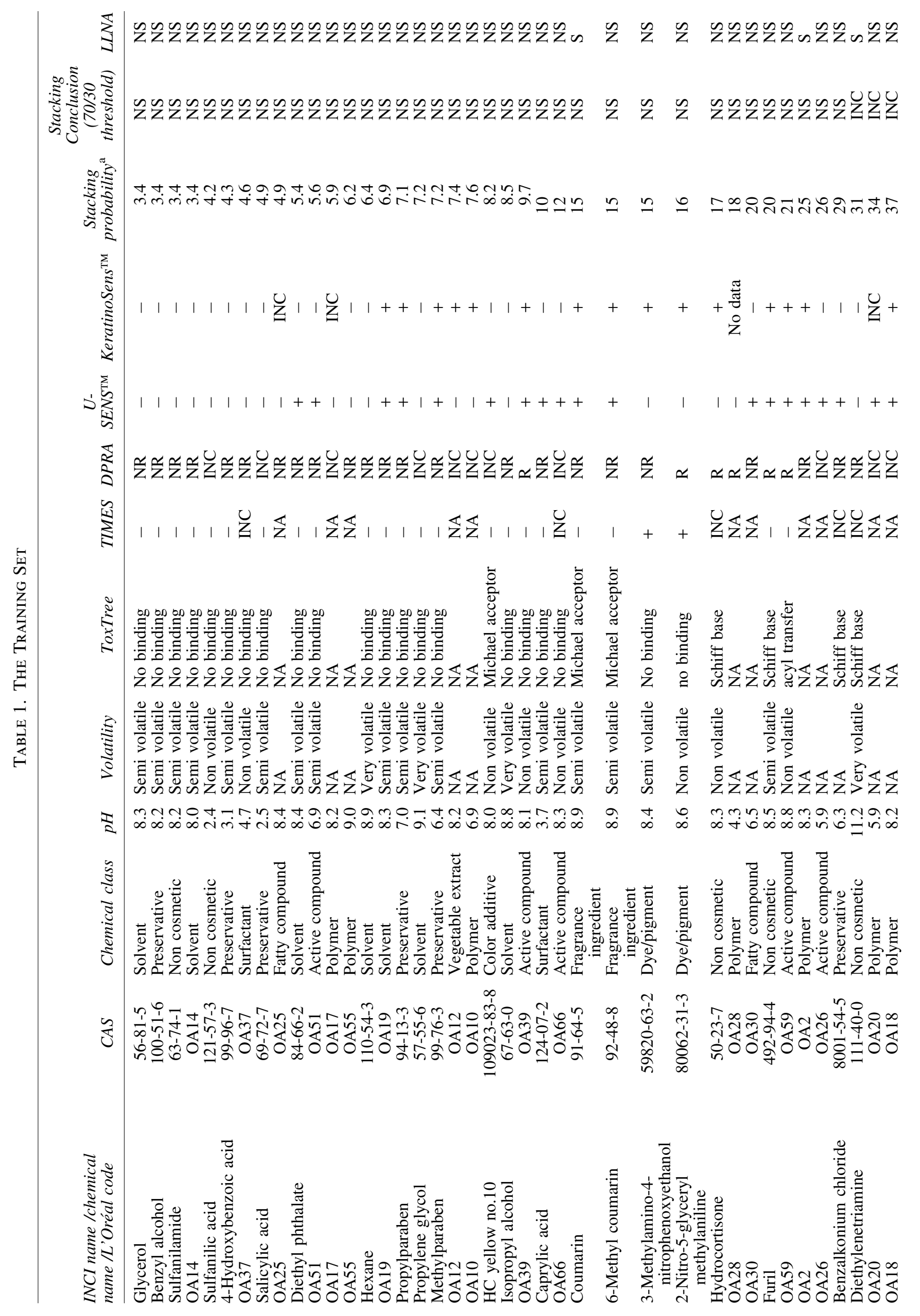




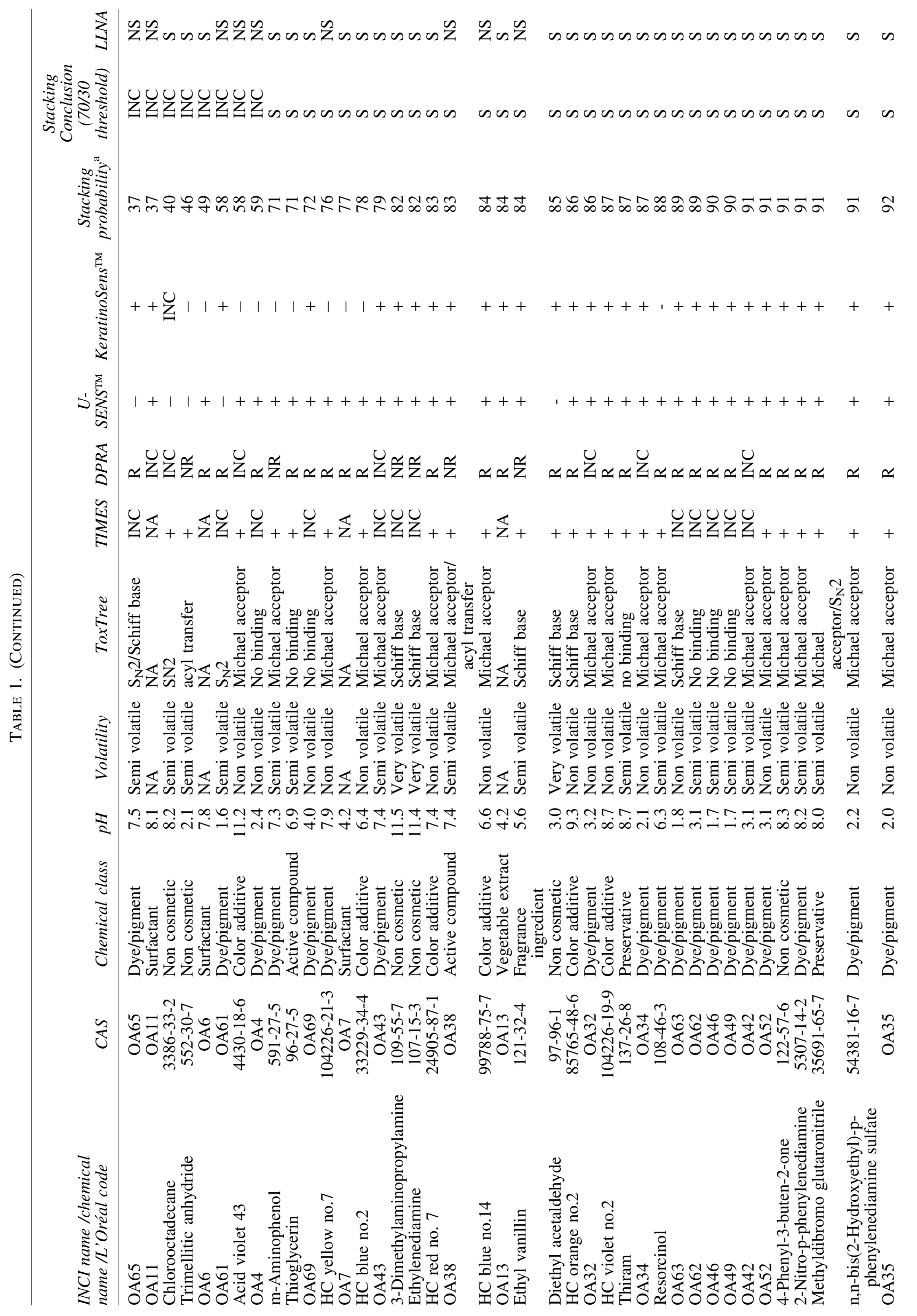




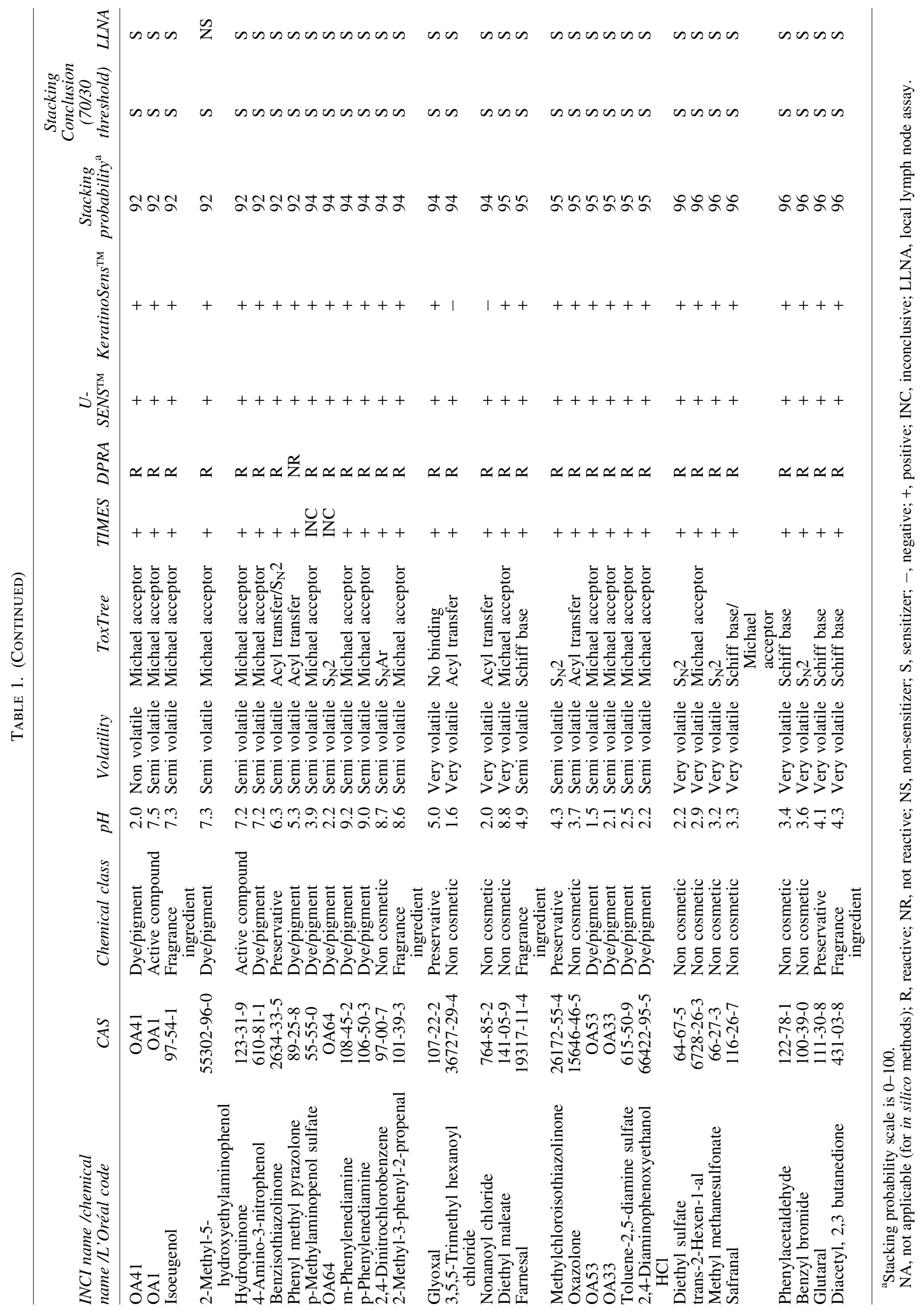




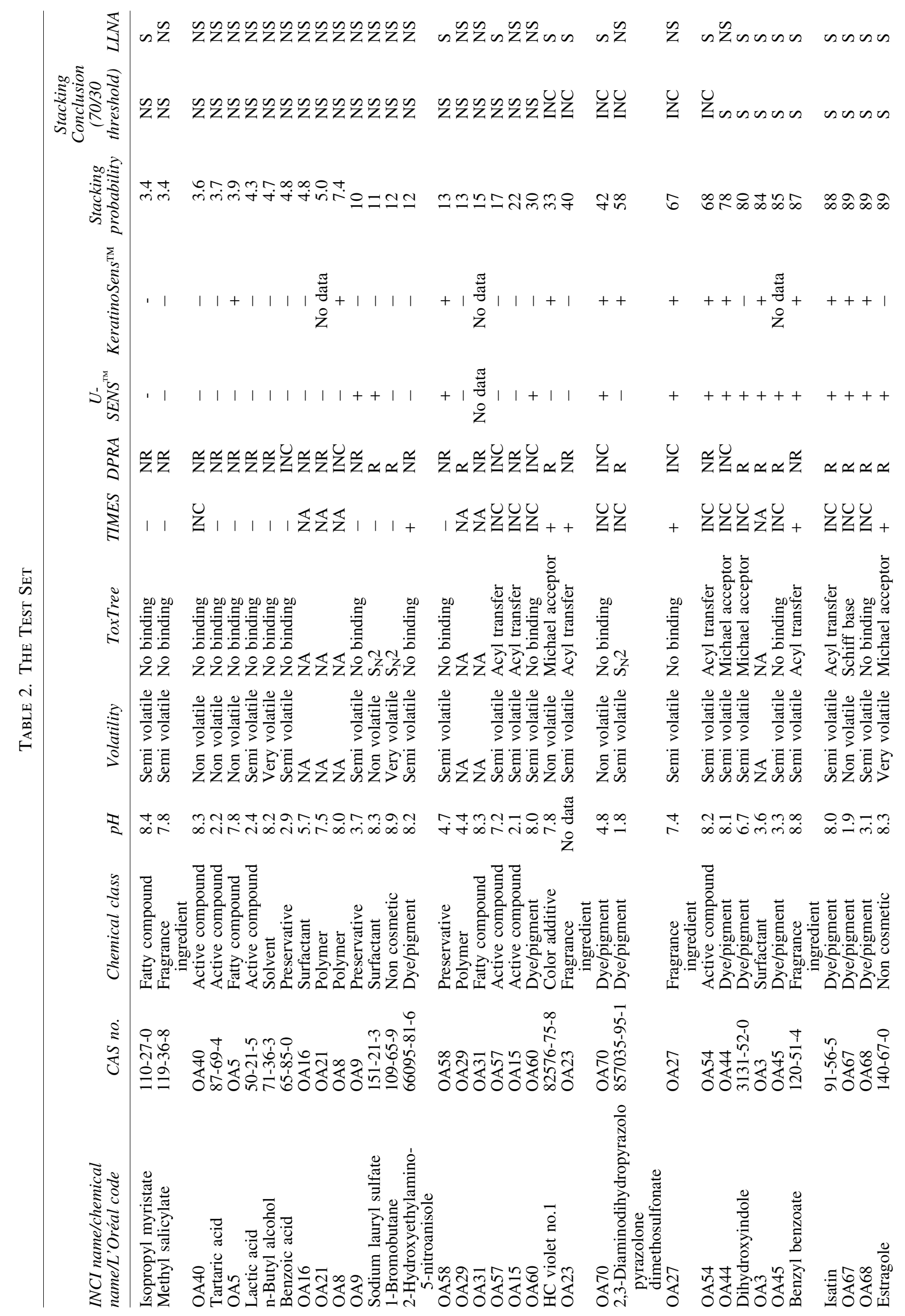




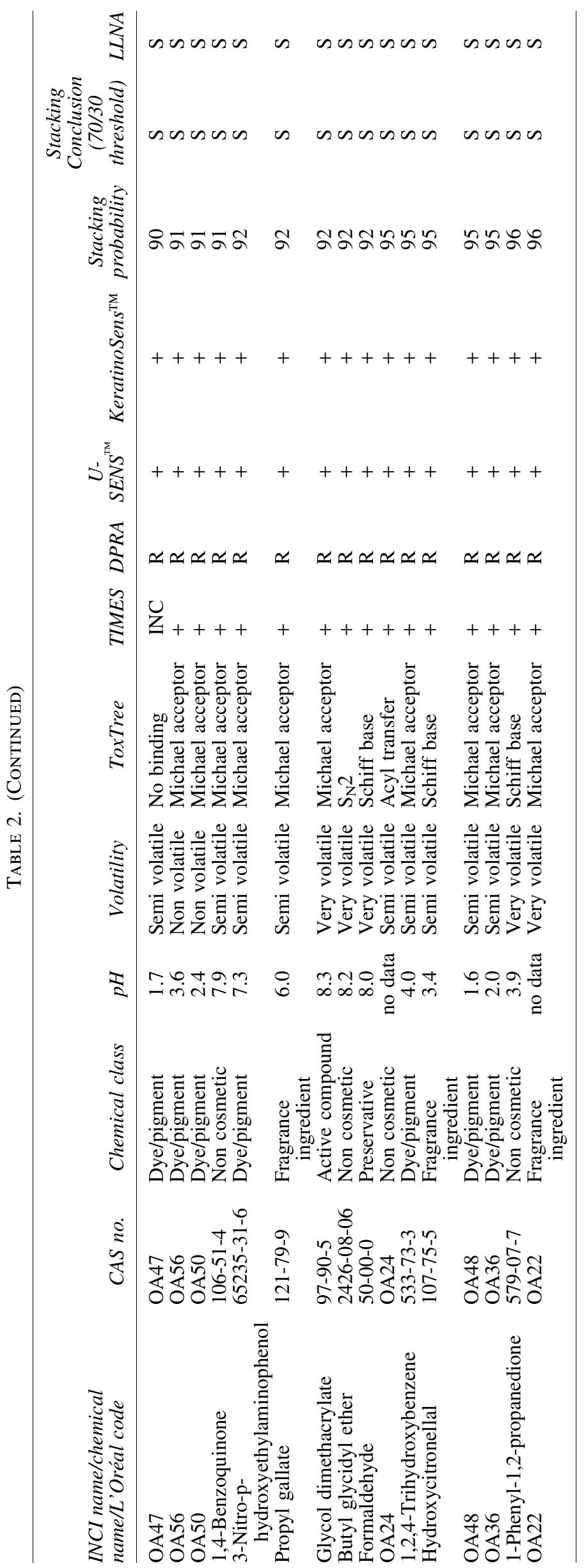


A

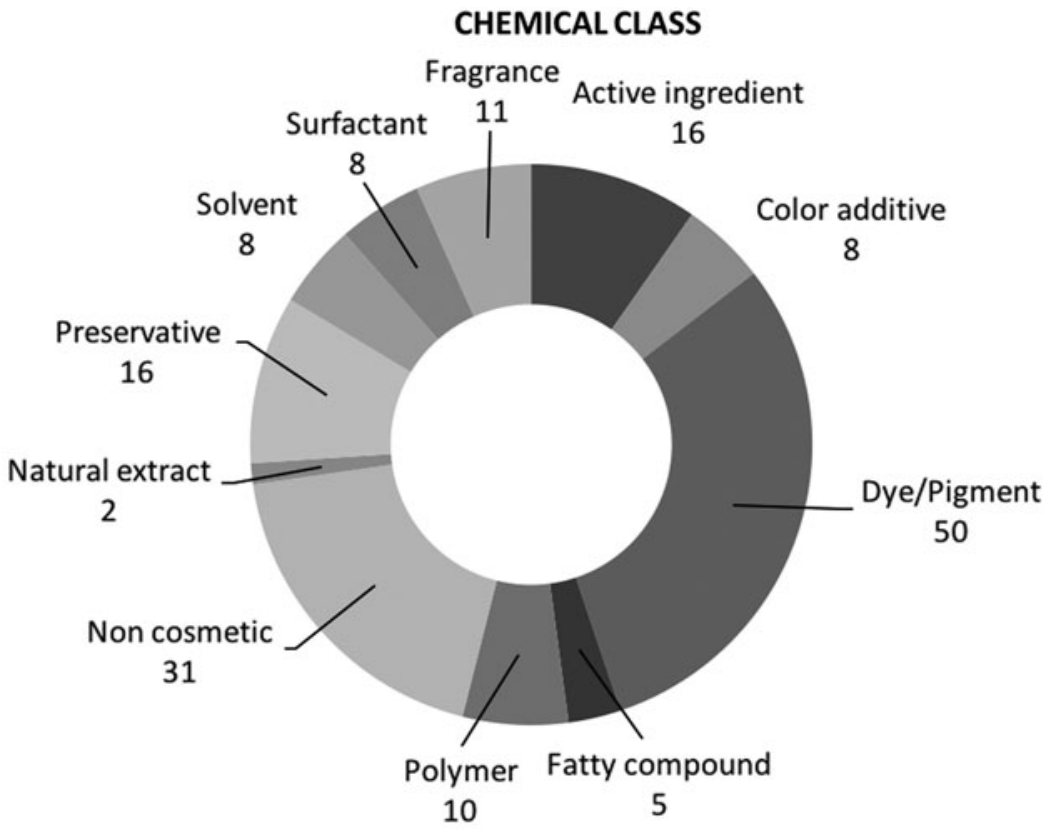

B

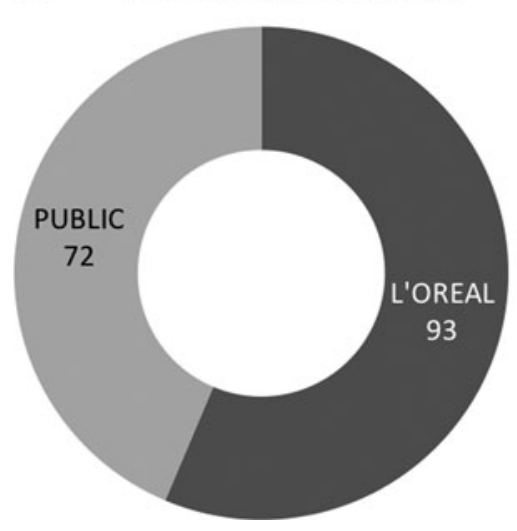

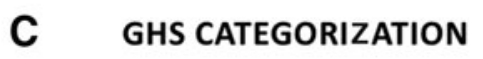

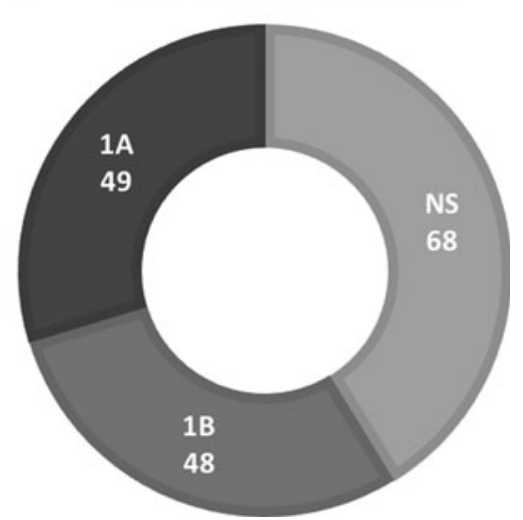

FIG. 1. Displays information concerning the combined test and training set of chemicals. (A) "chemical class" denotes the typical use of the substances, particularly with respect to cosmetic products, (B) provides the source of the LLNA data, and $(\mathbf{C})$ portrays the distribution of these substances into the three regulatory categories for skin sensitization designated by the UN GHS. LLNA, local lymph node assay. in skin cells in response to sensitizers ${ }^{31,42,43}$ and, in particular, in keratinocytes. In brief, HaCaT Nrf2-luciferase reporter cells are exposed to concentrations of the test chemical for 48 hours. A positive prediction occurs when the $\mathrm{I}_{\max }$ (maximal gene induction) is statistically significantly higher than $1.5 \times$ basal luciferase activity and the estimated concentration (EC) 1.5 value is below $1000 \mu \mathrm{M}$ in at least two out of the three repetitions. In addition, at the lowest concentration with a gene induction above 1.5-fold, the cellular viability should be above $70 \%$ and the dose response for luciferase induction should be similar between the repetitions. If these conditions are not met, the prediction normally is negative except in the specific situation where a sufficiently high concentration could not be tested, in which case the result is inconclusive. ${ }^{16}$

Key event three of the AOP was assessed using the USENS assay. ${ }^{44}$ This U937 activation test models the maturation of dendritic cells (DCs), which then activate naive $\mathrm{T}$ cells. The cell surface marker CD86, one of the classical markers of DC maturation, binds to molecules on T cell surface and is key in the T cell priming process. U-SENS measures the induction of CD86 in U937 cells, which serve as a surrogate for DCs. ${ }^{42}$ The individual conclusion of a U-SENS run is considered negative if the stimulation index (SI) of
CD86 is less than $150 \%$ at all non-cytotoxic concentrations (cell viability $\geq 70 \%$ ) and if no interference (solubility, color, or cytotoxicity) is observed. In all other cases, an SI of CD86 higher or equal to $150 \%$ and/or if interference is observed, the individual conclusion of a U-SENS run is considered positive. The final prediction is based on the majority concordant results. ${ }^{18,19}$ This simplified prediction model was peer reviewed by EURL ECVAM. The U-SENS underwent an industry-led validation study designed primarily to address the reproducibility of the method [Within Laboratory Reproducibility (WLR) and Between Laboratory Reproducibility (BLR)]. The WLR assessed in four laboratories on the basis of concordant classifications for 15 chemicals was around $90 \%$. The between-laboratory reproducibility was $84 \%(n=38)$. The accuracy of the method in discriminating between sensitizers and nonsensitizers (LLNA classifications) was $93 \%$ (sensitivity $99 \%$ and specificity $88 \%$ ) with the chemical tested in the validation study $(n=38)$. In addition, predictive capacities (toward LLNA in vivo data) on a larger set of 166 substances tested in-house were provided, indicating an accuracy of $86 \%$ (sensitivity $91 \%$ and specificity $65 \%$ ) against LLNA data. This binary prediction from the U-SENS assay was used in the model detailed below. 


\section{Data analysis}

Prediction methods for binary outcome belong to the wide set of supervised classification techniques. In this article, five methods representing five broad families of statistical models were selected (Boosting, Naïve Bayes, Support Vector Machines, Sparse Partial Least Squares Discriminant Analysis, and Expert Scoring); although very different, all these methods provide binary membership probabilities. Each method has its own variable selection based on a split into three subsamples: learning, test, and validation. To avoid any bias (nonpertinent decision rules) that may be induced by a particular choice of subsets, the splitting procedure is repeated several times (six times for this particular piece of work). The final selection then comprised the variables that were selected in all subsets (full details relating to this process has already been published ${ }^{34,35}$ ).

Stacking is known to be a successful way of linearly combining several models. ${ }^{45,46}$ The global stacking meta-model used for this work was constructed with the complete learning set and was prepared using the seven input variables detailed above ( $\mathrm{pH}$, volatility, ToxTree, TIMES-SS, DPRA, KeratinoSens, and U-SENS). These were entered into the model where they were run in the five different supervised classification models (Boosting, Naïve Bayes, Support Vector Machines, Sparse Partial Least Squares Discriminant Analysis, and Expert Scoring), each providing a probability of being a sensitizer. Details of each of these techniques have already been presented elsewhere. ${ }^{24,34,35}$ The intermediate probabilities delivered by each of the five methods, which are expected to be highly positively correlated (see Urbisch et al. ${ }^{33}$ ), are then used in the stacking meta-model to provide a final probability that a substance is a sensitizer, following the general principles already published. ${ }^{45,46}$ An open software of our stacking model is available in the Supplementary Data (Supplementary Data are available online at www.liebertpub.com/aivt).

\section{Results}

As detailed in Tables 1 and 2, the substances chosen for this study contained all reactive classes determined in silico with ToxTree, SNAr, SN2, Acyl transfer agent, Michel acceptor, and Shiff base formation; the wide range of measured $\mathrm{pH}$ values (from 1.5 to 11.5 ) indicates that the set contained a wide variety of acids, bases, and neutral chemicals. There was also a good spread of volatility, but note that to obtain an equilibrated set for the stacking meta-model purpose, the two Spicer classes "volatile" and "very volatile" were grouped together into one "very volatile" class. In the set, all three classes predicted in silico with Times are represented, with the weak and strong sensitizers grouped into "sensitizers" in the stacking meta-model.

A comprehensive overview of the results for the 113 substances used as the training set for this work is presented in Table 1. This set includes 47 substances identified as negative in the LLNA and 66 that were reported to be positive. LLNA results were derived from the database publications $^{47,48}$ supplemented by historical unpublished data on proprietary substances within the L'Oréal Laboratories database. The training set was used to derive prediction probabilities in each of the five individual models. The relative weights (contribution) of these five models are shown in
Table 3. Relative Contributions of the Five Models to the Stacking Meta-Model

\begin{tabular}{lc}
\hline Model & Relative weighting \\
\hline Boosting & 1.26 \\
Expert score & 2.05 \\
Sparse PLSDA & 1.16 \\
SVM & 1.2 \\
Bayes naïve & 1.34 \\
\hline
\end{tabular}

Table 3. These were then subjected to the stacking analysis to provide an overall probability of being a skin sensitizer. A key benefit of this is that the distribution of the probabilities provided by stacking becomes more bimodal than any of the other models individually, with the consequence that it leads to clearer conclusions for a greater number of chemicals. The probability thresholds to discriminate sensitizers from nonsensitizers can be varied to optimize the balance between predictivity (Kappa) and rate of inconclusive calls (INC) using the seven input variables (Fig. 2). As can be observed on the graph, as the gap between the probability cutoffs is widened, the kappa value increases, but this also impacts the number of inconclusive results, which increase markedly. Accordingly, a balance has to be struck, with the $30 \% / 70 \%$ limits being adopted. Therefore, the optimal decision criteria of the model are defined below:

- Chemicals with probability to be sensitizer $\geq 70 \%$ are predicted "Sensitizer";

- Chemicals with probability to be sensitizer $\leq 30 \%$ are predicted "Nonsensitizer";

- Chemicals with probability between those two thresholds are predicted "INC."

With these thresholds, the increased performance of the stacking meta-model compared to the individual methods is clearly evidenced in the performance data shown in Table 2. Confidence (kappa) in the stacking meta-model used to integrate the data sources proved to be very high

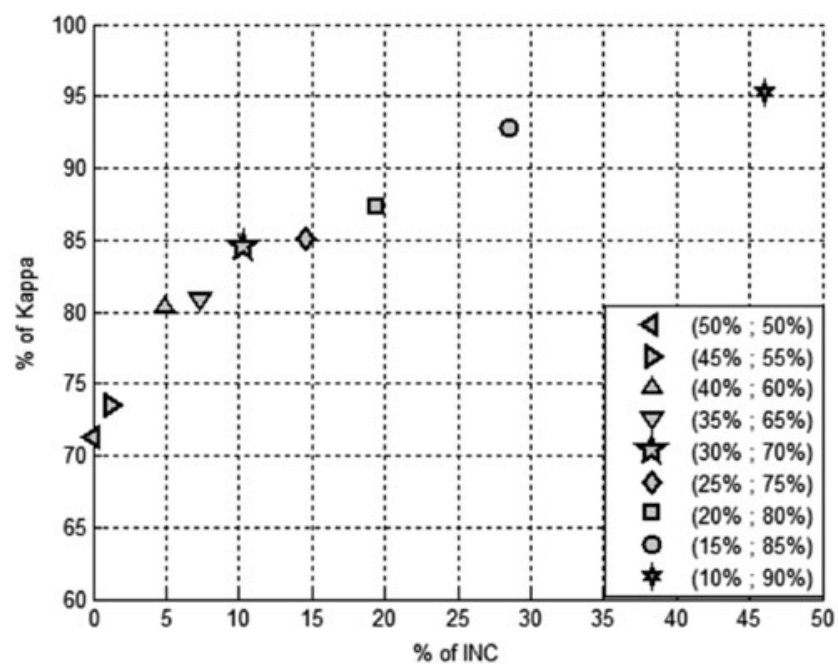

FIG. 2. Displays the behavior of the stacking meta-model (\% of Kappa and \% of Inconclusive data) using different cutoff options. 
(Fig. 2), and its overall accuracy was better than those from the five individual machine learning approaches, confirming that the stacking meta-model minimized the potential bias of individual statistical models and therefore the uncertainty linked to the structure of the prediction model (data not shown).

For the training set of substances, with the probability cutoffs set at $70 \%$ and $30 \%$, the predictive sensitivity was $97 \%$, specificity was $88 \%$, the overall accuracy was $93 \%$, and kappa was $85 \%$. Inconclusive outcomes occurred for 11 substances (4 sensitizers and 7 nonsensitizers) (Table 1). Figure 3 presents the outputs of the stacking meta-model in graphical form.

A further 52 substances (31 sensitizers and 21 nonsensitizers according to the LLNA) were subsequently assessed to test the effectiveness of the model developed with the training set of chemicals. All the results used to feed the model and the outcome of the final predictions for these 52 substances are presented in Table 2. The LLNA results were obtained from the same sources as those used for the training set. Figure 4 presents the outputs of the stacking meta-model in graphical form. The predictive sensitivity was $89 \%$, the specificity $95 \%$, the overall accuracy was $91 \%$, and kappa was $82 \%$, an outcome very much in line with the training set (Table 4). Also, a similar proportion of substances $(n=6)$ was deemed inconclusive (Table 2$)$.

Across both sets, 17 substances fell into the inconclusive category, resulting from a lack of sufficient data or conflicting datasets. There was not a dominant functional group associated with these, nor physicochemical properties (MW, cLogP, aand volatile class) or skin sensitization reactivity binding class $\left(\mathrm{S}_{\mathrm{N}} 2\right.$, Schiff base formation, acyl transfer agent, and Michael acceptor). Neither were there any dominant in vivo drivers of classification for INC (eight sensitizers and nine nonsensitizers in the LLNA). For any of these substances and depending upon their functional importance, it might be appropriate to consider the conduct of further in vitro or in silico work, perhaps together with the application of expert judgment, to clarify their skin sensitization potential. Indeed, a similar strategy could be appropriate for the characterization of any positive substance.

If the training and test datasets were combined, 148 substances in total with the 17 inconclusives excluded, the stacking meta-model offered an overall predictive accuracy of $93 \%$. Despite this high performance, a few substances were clear "outliers," for example, isopropyl myristate, coumarins, and three proprietary chemicals, OA2, OA57, and OA58. These will be considered in more detail in the discussion.

\section{Discussion}

The evolution from in vivo-based toxicological evaluation to predictions based entirely on in vitro and in silico information presents a number of intellectual and practical challenges. Not least among these is how to combine multiple data inputs into a single coherent conclusion. For skin sensitization, one effective strategy could be to adopt a minimalist approach using as little as the results from two non-animal assays, where, unless both results are negative, the substance is regarded as a classifiable sensitizer. ${ }^{49}$ Similar approaches involving three assays have been more widely promoted. ${ }^{11,29,50,51}$ All of these offer a level of predictive performance for regulatory decision-making, ${ }^{52}$ which is similar to that of in vivo methods that preceded them, in other words, in the region of $85 \%$. Interestingly, in terms of

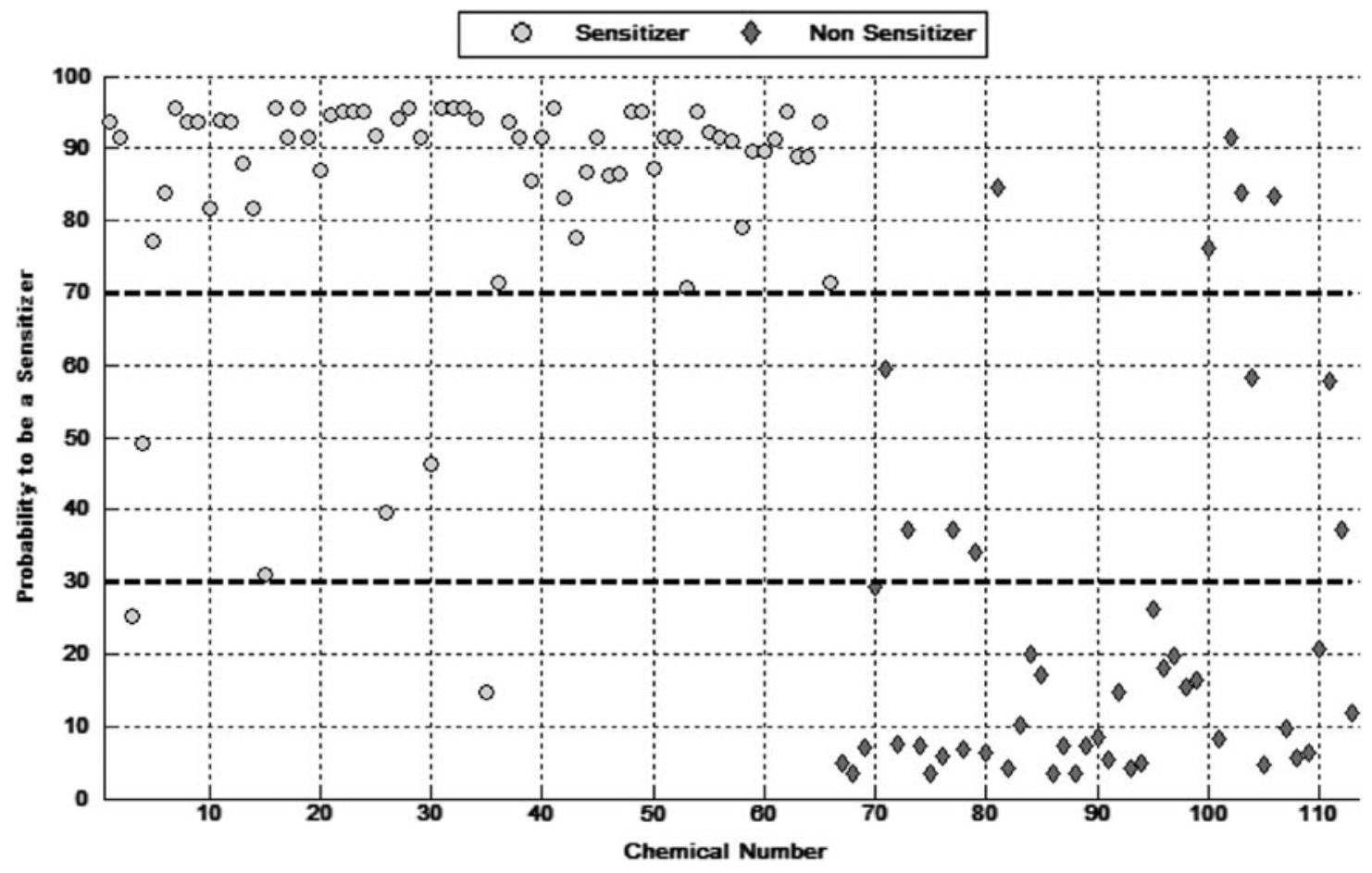

FIG. 3. Displays in graphical form the distribution of final probabilities in the stacking meta-model for the training set of 113 substances, with in vivo skin sensitizers shown as "circles" and in vivo nonsensitizers presented as "diamonds." 


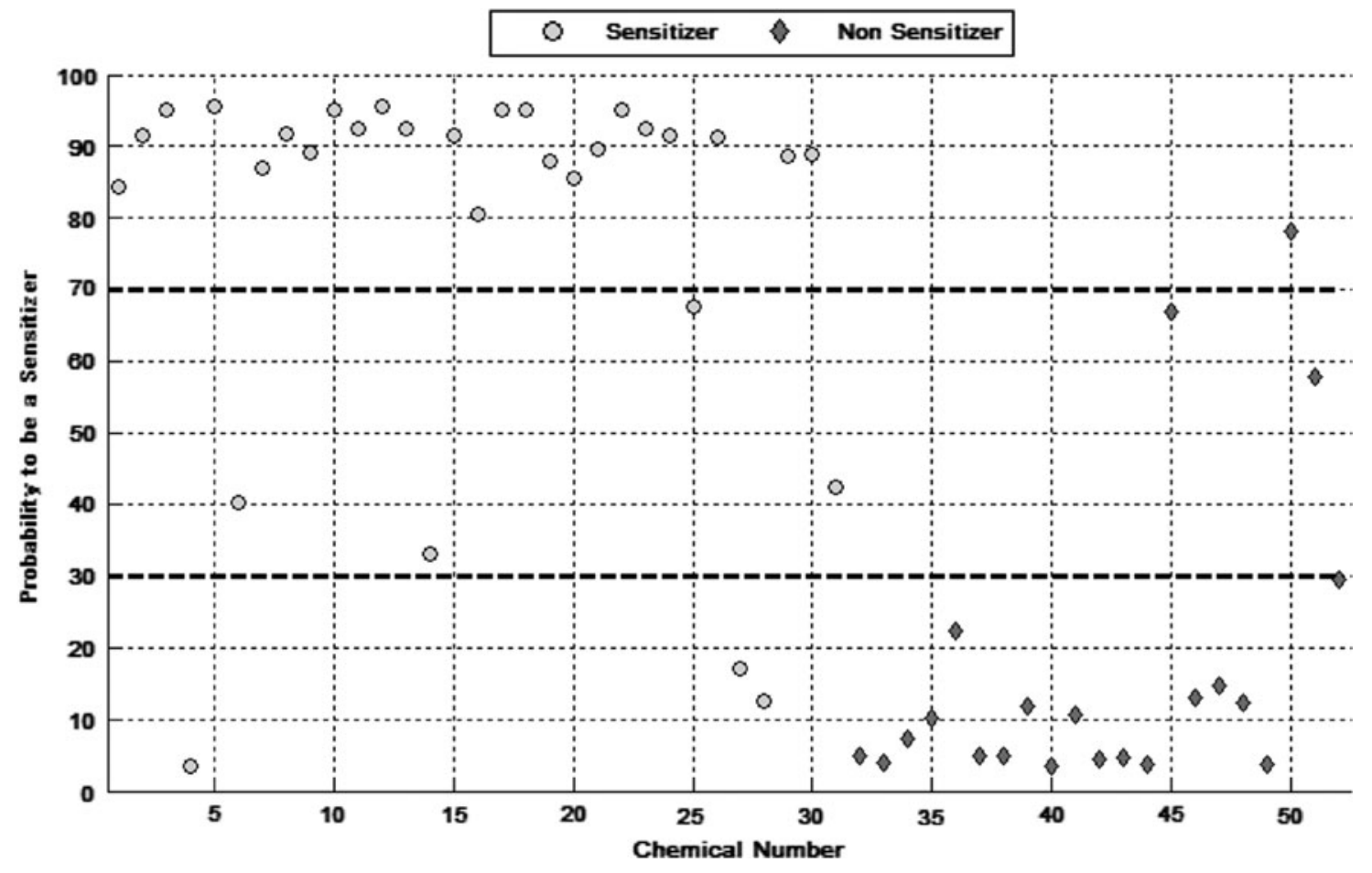

FIG. 4. Presents the individual probability to be a skin sensitizer for each substance of the test set of substances given by the stacking meta-model.

basic hazard identification for such regulatory classification, more complex statistical analyses and neural network approaches appear to perform at a fairly similar level. ${ }^{27,53}$

For consumers using cosmetic products, the avoidance of skin allergy is an important and topical health protection end point and goes well beyond the basic requirements of regulatory toxicology. In this regard, the work reported here is directed toward gaining an increased degree of confidence in the predictions from in vitro and in silico methods. The application of seven data inputs to five decision models, followed by application of a statistically based stacking meta-model, de- livered an analysis that markedly enhanced both sensitivity and specificity, with an overall accuracy in excess of $90 \%$ (Table 4). As noted earlier, for substances falling into the inconclusive category, the conduct of additional assessments could be considered a means to provide greater clarity, but this is highly likely to be on a case by case basis.

Notwithstanding the above, improving confidence in the predictions from non-animal testing also requires that discrepancies are examined carefully. For example, in this test set of 52 substances, the first chemical in the list is isopropyl myristate, rightly regarded as a false positive in the LLNA,

Table 4. Performance of In Silico and IN Vitro Methods Compared to Stacking Meta-Model

\begin{tabular}{|c|c|c|c|c|c|c|c|}
\hline & No data & $N A$ & $I N C$ & $\begin{array}{l}\text { Number of predicted } \\
\text { chemicals/number of } \\
\text { tested chemicals }\end{array}$ & Sensitivity $(\%)$ & Specificity $^{\mathrm{a}}(\%)$ & Accuracy $^{\mathrm{a}}(\%)$ \\
\hline ToxTree & 0 & 21 & 0 & $144 / 165$ & 86 & 63 & 78 \\
\hline Times-SS & 0 & 21 & 33 & $111 / 165$ & 96 & 75 & 88 \\
\hline DPRA & 0 & 0 & 25 & $140 / 165$ & 86 & 68 & 79 \\
\hline $\mathrm{U}-S E N S^{\mathrm{TM}}$ & 1 & 0 & 0 & $164 / 164$ & 92 & 55 & 77 \\
\hline KeratinoSens $^{\mathrm{TM}}$ & 4 & 0 & 4 & $157 / 161$ & 84 & 60 & 75 \\
\hline \multicolumn{8}{|l|}{ Stacking model } \\
\hline Learning set & 0 & 0 & 11 & $102 / 113$ & 97 & 88 & 93 \\
\hline Test set & 0 & 0 & 6 & $46 / 52$ & 89 & 95 & 91 \\
\hline Total set & 0 & 0 & 17 & $148 / 165$ & 94 & 90 & 93 \\
\hline
\end{tabular}

The table displays individual performance characteristics for the five inputs to the stacking meta-model to facilitate comparison with the performance of that model.

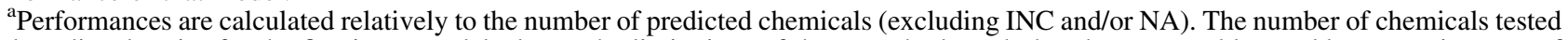
and predicted varies for the five input models due to the limitations of those methods such that they are unable to address certain types of chemistry (see Tables 1 and 3 for individual results). It is important to note that the performances are defined from different chemical set, therefore no strict comparison should be made. 
but which is confidently predicted as nonsensitizing by the stacking meta-model. This substance is an extremely rare sensitizer: probably the first case was in a feminine hygiene spray. ${ }^{54}$ Over three decades later, a German review concluded that it was an "extremely weak sensitizer....or possibly not a sensitizer at all". 55 This situation remains unchanged; isopropyl myristate likely represents a useful benchmark substance, being at the borderline of what can be detected clinically.

Next in the list is Coumarin, weakly positive at times in the LLNA, but a clear negative in the stacking meta-model. While this substance was often regarded as a very uncommon fragrance allergen, ${ }^{56}$ more recent information has indicated an upturn in the frequency of positive reactions to coumarin. ${ }^{57}$ However, the issue is complex: ToxTree indicates the substance is a Michael acceptor, yet both TIMES-SS and the DPRA are negative; in contrast, KeratinoSens and U-SENS are positive. Perhaps the key factor here is the purity of the material and the possible presence of other impurities. Thus, while a standard sample of coumarin was a weak skin sensitizer in the LLNA, the highly purified substance (99.9\%) was nonsensitizing. ${ }^{58-60}$ It is reasonable to conclude that coumarin itself is not a sensitizer and should not be identified as such in the stacking meta-model.

The remaining three discordant results were all substances proprietary to L'Oréal Laboratories. OA57 and OA58 are two examples of apparent false negatives. OA57 is an acyl transfer agent, on which basis alone, some evidence of skin sensitization might be anticipated. Surprisingly then, negative results were obtained in U-SENS and KeratinoSens. However, the LLNA was positive with an EC3 value of 17\%; so it would be reasonable to regard OA57 as a weak sensitizer. In contrast, OA58 is a linear structure organic chemical without any positive predictions from ToxTree, TIMES-SS, or DPRA. Nevertheless, it gave positive results in U-SENS and KeratinoSens and proved to be a moderate sensitizer in the LLNA. In the absence of any human data, it seems appropriate to conclude that OA58 is a sensitizer and thus a false negative in the stacking meta-model.

OA2 is a complex polymeric material, such that none of the predictions based on chemistry/reactivity could be applied. It had proven to be a borderline positive in the LLNA (EC3 value of $83 \%$ ), but with positive results in U-SENS and KeratinoSens. However, a human repeated insult patch test (HRIPT) of OA2 carried out at a concentration of $40 \%$ was negative (L'Oréal, unpublished data). In the absence of any further information, OA2 might easily be concluded to be a false negative, but the fact that it was barely positive in the LLNA and failed to cause any effect at a very high concentration in a human test likely tips the balance in favor of the negative prediction, from the stacking meta-model being the most appropriate.

As the title of this article indicates, the purpose of the work reported herein is the development of a non-animal approach that will optimize the assurance of consumer safety for the skin sensitization end point. When an ingredient has been identified as a potential skin sensitizer, the assessment of risk normally requires measurement of its relative potency to complete the safety evaluation process. ${ }^{61,62}$ Current validated nonanimal approaches remain somewhat deficient in that respect, although it must be recognized that considerable efforts are underway to address this problem..$^{22,27,53,63,64}$ For identified skin sensitizers, this current absence of potency data means that strategies such as quantitative risk assessment (QRA) are difficult to employ, leaving only considerations such as the threshold of toxicological concern (TTC), which has only a limited area of applicability to very low use concentrations, as a way forward. ${ }^{65,66}$ In contrast, nonsensitizing ingredients need not be subject to any risk assessment/ management (for this toxicology end point) and they may therefore be used at any level in a product. Consequently, it is essential to have a high degree of confidence in the identification of nonsensitizing materials. Non-animal testing strategies orientated toward regulatory acceptance for chemicals testing (such as REACH) commonly aim to identify sensitizers, and so usually enhance sensitivity, at the expense of specificity. ${ }^{29,67,68}$ By application of the most commonly used strategy, often referred to as the " 2 out of 3 " method, ${ }^{29,31,50}$ this dataset delivers a sensitivity of $93 \%$, but with a specificity of only $59 \%$ due to the relatively high proportion of false positives. However, in the work reported here, while retaining a high sensitivity in terms of the accurate identification of skin sensitizing chemicals, confidence that an ingredient is truly non-sensitizing (specificity) has been increased to $90 \%$.

In conclusion, the stacking meta-model reported in this article capitalizes on multiple inputs from seven individual predictions to deliver improved performance and therefore enhanced confidence in the discrimination of skin sensitizers from nonsensitizers. It is suggested that the key remaining gap in this toxicology domain, the prediction of skin sensitization potency, may benefit from a similar approach, maximizing the use of evidence from individual strands of prediction, while minimizing the impact of the limitations from any particular one of them.

\section{Author Disclosure Statement}

DAB was compensated by L'Oréal for the time spent in the preparation and review of this article; all other authors are fully paid employees of the L'Oréal company.

\section{References}

1. Russell WMS, Burch RL. The Principles of Human Experimental Technique. London, UK: Methuen, 2016.

2. EU 2010. Directive 2010/63/EU of the Parliament and Council of 22 September 2010 on the protection of animals used for scientific purposes. http://eur-lex.europa.eu/legalcontent/EN/TXT/?uri=CELEX:32010L0063 (last accessed, Oct. 16, 2016).

3. EU. Directive 2003/15/EC of the European parliament and the council of 27 February 2003 amending council directive $76 / 768 / \mathrm{EEC}$ on the approximation of the laws of the members states relating to cosmetic products. Off J Eur Union 2003:L66;26-35.

4. EU. Regulation (EC) No 1223/2009 of the European Parliament and of the Council of 30 November 2009 on Cosmetic Products. http://eur-lex.europa.eu/legal-content/EN/ ALL/?uri=CELEX\% 3A32009R1223 (last accessed Apr. 28, 2016).

5. Basketter DA, White IR. Legislative aspects of cosmetic safety in the European Union. Cosmetics 2016:3;17-24.

6. Adler S, Basketter DA, Creton S, et al. Alternative (nonanimal) methods for cosmetics testing: current status and future prospects-2010. Arch Toxicol 2011:85;367-485. 
7. Leist M, Hasiwa N, Rovida C, et al. Consensus report on the future of animal-free systemic toxicity testing. ALTEX 2014:31;341-356.

8. Andersen KE, Maibach HI. Contact Allergy: Predictive test in guinea pigs. Current Problems in Dermatology. Basel: Karger, 1985.

9. Botham PA, Basketter DA, Maurer Th, et al. Skin sensitization - a critical review of predictive test methods in animal and man. Food Chem Toxicol 1991:29;275-286.

10. Gerberick GF, Ryan CA, Kimber I, et al. Local lymph node assay validation assessment for regulatory purposes. Am J Contact Dermat 2000:11;3-18.

11. Basketter DA, Ashikaga T, Casati S, et al. Alternatives for Skin sensitisation testing and assessment. Regul Toxicol Pharmacol 2015:73;660-666.

12. Reisinger K, Hoffmann S, Alépée N, et al. Systematic evaluation of non-animal test methods for skin sensitisation safety assessment. Toxicol In Vitro 2015:29;259-270.

13. OECD 2012. The Adverse Outcome Pathway for Skin Sensitisation Initiated by Covalent Binding to Proteins. Part 1: Scientific Evidence. Series on Testing and Assessment No.168. http://www.keepeek.com/Digital-Asset-Management/oecd/ environment/the-adverse-outcome-pathway-for-skinsensitisation-initiated-by-covalent-binding-to-proteins 9789264221444-en\#page1 (last accessed December 8, 2017).

14. OECD 2012. The Adverse Outcome Pathway for Skin Sensitisation Initiated by Covalent Binding to Proteins. Part 2: Use of the AOP to Develop Chemical Categories and Integrated Assessment and Testing Approaches. Series on Testing and Assessment. No. 168. http://www.oecd.org/officialdocuments/ publicdisplaydocumentpdf $/$ ?cote $=$ env $/ \mathrm{jm} / \operatorname{mono}(2012) 10 /$ part2\&doclanguage $=$ en (last accessed December 8, 2017).

15. OECD 2015. OECD Guideline for Testing of Chemicals No. 442C: In Chemico Skin Sensitisation: Direct Peptide Reactivity Assay (DPRA). Paris, France.

16. OECD 2015. OECD Guideline for Testing of Chemicals No. 442D: In Vitro Skin Sensitisation: ARE-Nrf2 Luciferase Test Method. Paris, France.

17. OECD 2015. OECD Draft Proposal for a new Test Guideline: In Vitro Skin Sensitisation: Human Cell Line Activation Test (h-CLAT). Paris, France. www.oecd.org/env/ehs/ testing/151216-Draft-h-CLAT-TG-After-Expert-Meeting(clean)-Final.pdf (last accessed Mar. 02, 2016).

18. Piroird C, Ovigne JM, Rousset F, et al. The myeloid U937 skin sensitization test (U-SENS) to address the activation of dendritic cell event in the adverse outcome pathway for skin sensitization. Toxicol In Vitro 2014:29;901-916.

19. Alépée N, Piroird C, Aujoulat M, et al. Prospective multicentre study of the U-SENS test method for skin sensitization testing. Toxicol In Vitro 2015:30;373-382.

20. Kimura Y, Fujimura C, Ito Y, et al. Optimization of the IL-8 Luc assay as an in vitro test for skin sensitization. Toxicol In Vitro 2015:29;1816-1830.

21. Ramirez T, Stein N, Aumann A, et al. Intra- and interlaboratory reproducibility and accuracy of the LuSens assay: a reporter gene-cell line to detect keratinocyte activation by skin sensitizers. Toxicol In Vitro 2016:32;278-286.

22. Cottrez F, Boitel E, Ourlin JC, et al. SENS-IS, a 3D reconstituted epidermis based model for quantifying chemical sensitization potency: reproducibility and predictivity results from an inter-laboratory study. Toxicol In Vitro 2016: 32;248-260.

23. Patlewicz G, Kuseva C, Kesova A, et al. Towards AOP application-implementation of an integrated approach to testing and assessment (IATA) into a pipeline tool for skin sensitization. Regul Toxicol Pharmacol 2014:69;529-545.

24. OECD 2016. Guidance on Reporting of Defined Approaches in Integrated Approaches to Testing and Assessment (IATA) and 12 Skin Sensitisation Case Studies. See Annex 1. http:// www.oecd.org/officialdocuments/publicdisplaydocumentpdf/ ?cote=env/jm/mono(2016)29/ann1\&doclanguage=en (last accessed December 8, 2017).

25. Strickland J, Zang Q, Kleinstreuer N, et al. Integrated decision strategies for skin sensitization hazard. J Appl Toxicol 2016:36;1150-1162.

26. MacKay C, Davies M, Summerfield V, et al. From pathways to people: applying the adverse outcome pathway (AOP) for skin sensitization to risk assessment. ALTEX 2013:30; 473-486.

27. Jaworska JS, Natsch A, Ryan C, et al. Bayesian integrated testing strategy (ITS) for skin sensitization potency assessment: a decision support system for quantitative weight of evidence and adaptive testing strategy. Arch Toxicol 2015: 89;69-75.

28. Takenouchi O, Fukui S, Okamoto K, et al. Test battery with the human cell line activation test, direct peptide reactivity assay and DEREK based on a 139 chemical data set for predicting skin sensitizing potential and potency of chemicals. J Appl Toxicol 2015:35;1318-1332.

29. Urbisch D, Mehling A, Guth K, et al. Assessing skin sensitization hazard in mice and men using non-animal test methods. Regul Toxicol Pharmacol 2015:71;337-351.

30. Ezendam J, Braakhuis HM, Vandebriel RJ. State of the art in non-animal approaches for skin sensitization testing: from individual test methods towards testing strategies. Arch Toxicol 2016:90;2861-2863.

31. Natsch A, Ryan CA, Foertsch L, et al. A dataset on 145 chemicals tested in alternative assays for skin sensitization undergoing prevalidation. J Appl Toxicol 2013:33;13371352.

32. Urbisch D, Honarvar N, Kolle SN, et al. Peptide reactivity associated with skin sensitization: the QSAR Toolbox and TIMES compared to the DPRA. Toxicol In Vitro 2016:34; 194-203.

33. Gomes $\mathrm{CH}$, Nocairi $\mathrm{H}$, Thomas $\mathrm{M}$, et al. Stacking prediction for a binary outcome. COMPSTAT, 20th International Conference on Computational Statistics, Limassol, Cyprus, 2012, pp. 271-282.

34. Gomes $\mathrm{CH}$, Nocairi $\mathrm{H}$, Thomas $\mathrm{M}$, et al. A simple and robust scoring technique for binary classification. Artif Intell Res 2014:3;52-58.

35. Noçairi H, Gomes C, Thomas M, et al. Improving Stacking methodology for Combining classifiers: application to cosmetic industry. Electron J Appl Stat Anal 2016:09;340-361.

36. US EPA 2012. EPI SuiteTM, version 4.1. www.epa.gov/ sites/production/files/2015-05/documents/05.pdf (last accessed Aug. 30, 2017).

37. Spicer CW. Hazardous Pollutant Handbook, Measurements, Properties and Fate in Ambient Air. FL, USA: CRC Press LLC, 2002.

38. OECD 2013. OECD Guidelines for the Testing of Chemicals, Section 1 Physical-Chemical properties, Test No. 122: Determination of $\mathrm{pH}$, Acidity and Alkalinity. Paris, France: OECD publishings ISSN, pp. 2074-5753.

39. Aptula AO, Roberts DW. Mechanistic applicability domains for non-animal-based prediction of toxicological end points: general principles and application to reactive toxicity. Chem Res Toxicol 2006:19;1097-1105. 
40. Patlewicz G, Dimitrov SD, Low LK, et al. TIMES-SSa promising tool for the assessment of skin sensitization hazard. A characterization with respect to the OECD validation principles for (Q)SARs and an external evaluation for predictivity. Regul Toxicol Pharmacol 2007:48;225-239.

41. Gerberick GF, Vassallo JD, Bailey RE, et al. Development of a peptide reactivity assay for screening contact allergens. Toxicol Sci 2004:81;332-343.

42. Ade N, Martinozzi-Teissier S, Pallardy M, et al. Activation of U937 cells by contact sensitizers: CD86 expression is independent of apoptosis. J Immunotoxicol 2006:3;189-197.

43. Natsch A. The Nrf2-Keap1-ARE toxicity pathway as a cellular sensor for skin sensitizers-functional relevance and a hypothesis on innate reactions to skin sensitizers. Toxicol Sci 2010:113;284-292.

44. OECD 2016. U-SENS Draft Test Guideline. Paris, France: OECD.

45. Wolpert D. Stacked generalization. Neural Netw 1992:5; 241-259.

46. Breiman L. Stacked regressions. Machine Learn 1996:24; 49-64.

47. Gerberick GF, Ryan CA, Kern PS, et al. Compilation of historical local lymph node assay data for the evaluation of skin sensitization alternatives. Dermatitis 2005:16:157-202.

48. Kern PS, Gerberick GF, Ryan C, et al. Historical local lymph node data for the evaluation of skin sensitization alternatives: a second compilation. Dermatitis 2010:21;8-32.

49. Roberts DW, Patlewicz G. Non-animal assessment of skin sensitisation hazard: is an integrated testing strategy needed, and if so what should be integrated? J Appl Toxicol 2017: $38 ; 41-50$.

50. Bauch C, Kolle SN, Ramirez T, et al. Putting the parts together: combining in vitro methods to test for skin sensitizing potentials. Regul Toxicol Pharmacol 2012:63;489-504.

51. van der Veen JW, Rorije E, Emter R, et al. Evaluating the performance of integrated approaches for hazard identification of skin sensitizing chemicals. Regul Toxicol Pharmacol 2014:69;371-379.

52. United Nations 2015. Globally Harmonized System of Classification and Labelling of Chemicals (Sixth Revised Edition). https://www.unece.org/fileadmin/DAM/trans/danger/ publi/ghs/ghs_rev06/English/03e_part3.pdf (last accessed December 8, 2017).

53. Nukada Y, Miyazawa M, Kazutoshi S, et al. Data integration of non-animal tests for the development of a test battery to predict the skin sensitizing potential and potency of chemicals. Toxicol In Vitro 2013:27;609-618.

54. Fisher AA. Allergic reaction to feminine hygiene sprays. Arch Dermatol 1973:108;801-802.

55. Uter W, Schnuch A, Geier J, et al. Isopropyl myristate recommended for aimed rather than routine patch testing. Contact Dermatitis 2004:50;242-244.

56. Schnuch A, Uter W, Geier J, et al. Sensitization to 26 fragrances to be labelled according to current European regula- tion. Results of the IVDK and review of the literature. Contact Dermatitis 2007:57;1-10.

57. Krautheim A, Uter W, Frosch P, et al. Patch testing with fragrance mix II: results of the IVDK 2005-2008. Contact Dermatitis 2010:63;262-269.

58. Scientific Committee on Consumer Products 2006. Opinion on Coumarin. Adopted 20/06/2006. http://ec.europa.eu/health/ ph_risk/committees/04_sccp/docs/sccp_o_061.pdf (last accessed Nov. 24, 2016).

59. Vocanson M, Goujon C, Chabeau G, et al. The skin allergenic properties of chemicals may depend on contaminantsevidence from studies on coumarin. Int Arch Allergy Immunol 2006:140;231-238.

60. Vocanson M, Valeyrie M, Rozières A, et al. Lack of evidence for allergenic properties of coumarin in a fragrance allergy mouse model. Contact Dermatitis 2007:57; 361-364.

61. Api AM, Basketter DA, Cadby PA, et al. Dermal sensitization quantitative risk assessment (QRA) for fragrance ingredients. Regul Toxicol Pharmacol 2008:52;3-23.

62. Basketter DA. Skin sensitisation, adverse outcome pathways and alternatives. ATLA 2016:44;431-436.

63. Natsch A, Emter R, Gfeller H, et al. Predicting skin sensitizer potency based on in vitro data from KeratinoSens and kinetic peptide binding: global versus domain-based assessment. Toxicol Sci 2015:143;319-332.

64. Dimitrov S, Detroyer A, Piroird C, et al. Accounting for data variability, a key factor in in vivo/in vitro relationships: application to the skin sensitization potency (in vivo LLNA versus in vitro DPRA) example. J Appl Toxicol 2016:36; 1568-1578.

65. Safford RJ, Aptula AO, Gilmour N. Refinement of the Dermal Sensitisation Threshold (DST) approach using a larger dataset and incorporating mechanistic chemistry domains. Regul Toxicol Pharmacol 2011:60;218-224.

66. Safford RJ, Api AM, Roberts DW, et al. Extension of the Dermal Sensitisation Threshold (DST) approach to incorporate chemicals classified as reactive. Regul Toxicol Pharmacol 2015:72;694-701.

67. Macmillan DS, Canipa SJ, Chilton ML, et al. Predicting skin sensitisation using a decision tree integrated testing strategy with an in silico model and in chemico/in vitro assays. Regul Toxicol Pharmacol 2016:76;30-38.

68. Zang Q, Paris M, Lehmann D, et al. Prediction of skin sensitization potency using machine learning approaches. J Appl Toxicol 2017:37;792-805.

Address correspondence to: Dr. David Basketter

DABMEB Consultancy Ltd. Sharnbrook MK44 1PR United Kingdom

E-mail: dabmebconsultancyltd@me.com 